\title{
Esperando lo inesperado, la pandemia del milenio... hasta el momento: COVID-19
}

\section{Expecting the unexpected, the pandemic of the millennium... till now: COVID-19}

\author{
Alfonso J. Rodriguez-Morales ${ }^{1,2}$, Wilmer E. Villamil-Gómez ${ }^{2,3,4}$
}

\section{Señor Director:}

Al iniciar el milenio, dos décadas atrás, el mundo experimentó los primeros brotes epidémicos de gran preocupación, como fueron precisamente el Síndrome Respiratorio Agudo Severo (SARS) causado por el SARS$\mathrm{CoV}$, y la influenza aviar H5N1, ambos originados en Asia, siendo el primero declarado como una emergencia sanitaria de preocupación internacional (ESPI) por parte de la Organización Mundial de la Salud (OMS) $)^{(1-3)}$. Seis años más tarde, en 2009, de nuevo la influenza no estacional, en este caso porcina H1N1, originada en México, fue considerada no solo una ESPI, sino además declarada una pandemia el 11 de junio de ese año (y hasta el 10 de agosto de 2010), dado que causó 622,482 casos (con un estimado de 1,600,000), y 18,449 muertes, en los cinco continentes. América Latina no fue la excepción, con casos confirmados en la mayoría de los países de la región ${ }^{(4-6)}$. Brotes y epidemias de Polio, Ébola y Zika, entre 2014 a 2020, marcaron 5 declaraciones adicionales de ESPI, hasta el año 2020, en el cual la OMS no solo declaró ESPI a la epidemia de la enfermedad por Coronavirus 2019 (COVID-19), causada por el virus SARS-CoV-2, sino que días más tarde, también le declaró pandemia ${ }^{(7-15)}$.

Ciertamente, como con otras epidemias (como chikungunya y Zika), el mundo no esperaba directamente una pandemia ${ }^{(16)}$. Pero la OMS ya venía en un proceso de preparación para los coronavirus como SARS y MERS, que habían causado en 2002 y 2012, respectivamente epidemias de importancia, especialmente en Asia $^{(15,17)}$. Lamentablemente, nadie imaginó que al 9 de abril de 2020, se habrían reportado más de $1,480,000$ casos y más de 88,500 muertes $^{(18)}$. Para América Latina, más de 34,000 casos, con más de 31,000 casos en Sur América, a expensas principalmente de Brasil $(>13,000)$, y más de 3,200 en Centro América, la mayoría de Panamá $(>2,200)^{(19)}$. Hasta lo que va del milenio, se puede afirmar que esta

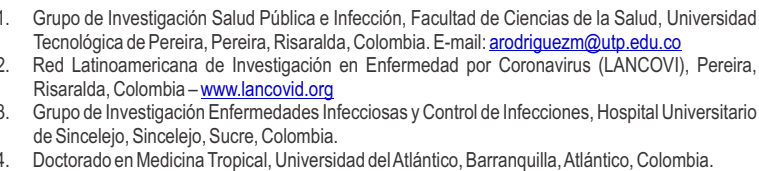

es la pandemia del milenio. Ninguna otra enfermedad infecciosa o epidemia, había logrado paralizar social y económicamente al mundo, detener los viajes internacionales ${ }^{(20,21)}$, el turismo, eventos deportivos mayores (como los Juegos Olímpicos de verano) ${ }^{(22)}$, cancelar la Semana Santa ${ }^{(23)}$, entre otros múltiples impactos a los cuales ha conllevado el cierre de países y las cuarentenas establecidas con el fin de reducir la transmisión.

En América Latina, muchísimos países se encuentran ya en fase de transmisión comunitaria ${ }^{(24)}$, y se han reportado más de 1,300 muertes (667 en Brasil). Para el caso de esta región prácticamente en un solo mes, dado que los primeros casos se reportaron a finales de Febrero 2020, primero en $\mathrm{Brasil}^{(25,26)}$. Una región, que de por sí, ya estaba siendo considerablemente afectada por epidemias de dengue, malaria, sarampión, tos ferina y difteria, entre otras, muchas de ellas propiciadas por la migración forzada desde Venezuela $^{(27-29)}$

La región requiere entender el impacto de las medidas, la importancia de mejorar la vigilancia epidemiológica ${ }^{(30)}$, incluyendo la genómica ${ }^{(31-34)}$, así como propiciar más investigación operativa que permita rápidamente entender no solo aspectos epidemiológicos y clínicos $^{(35-37)}$, sino como abordar terapéuticamente el COVID-19 de manera oportuna ${ }^{(38)}$. Tal y como lo ha desarrollado Colombia, los demás países en la región necesitan desarrollar sus propios consensos nacionales de atención basados en evidencias científicas, para orientar a los profesionales de la salud en el mejor manejo de los pacientes, a la par del conocimiento científico disponible ${ }^{(38)}$. La situación epidemiológica corresponde analizar diariamente, y observar en los próximos como se da el impacto de las medidas tomadas. Entre tanto, por ejemplo Perú y Colombia, experimentan un incremento considerable en el número de casos reportados, en su mayoría ya de transmisión comunitaria, pero aún con muchos casos importados, dado que aún no ha transcurrido tiempo suficiente para dejar de verlos (Figura $N^{\circ} 1$ ). Ambos países se encuentran ya reportando sobre 200 casos diarios, con acumulados por arriba de los 2,000 casos. 
Figura $N^{\circ} 1$. Comportamiento diario de casos confirmados de COVID-19 en Colombia y Perú, desde el primer día de introducción. Fuente: https://ourworldindata.org/coronavirus.

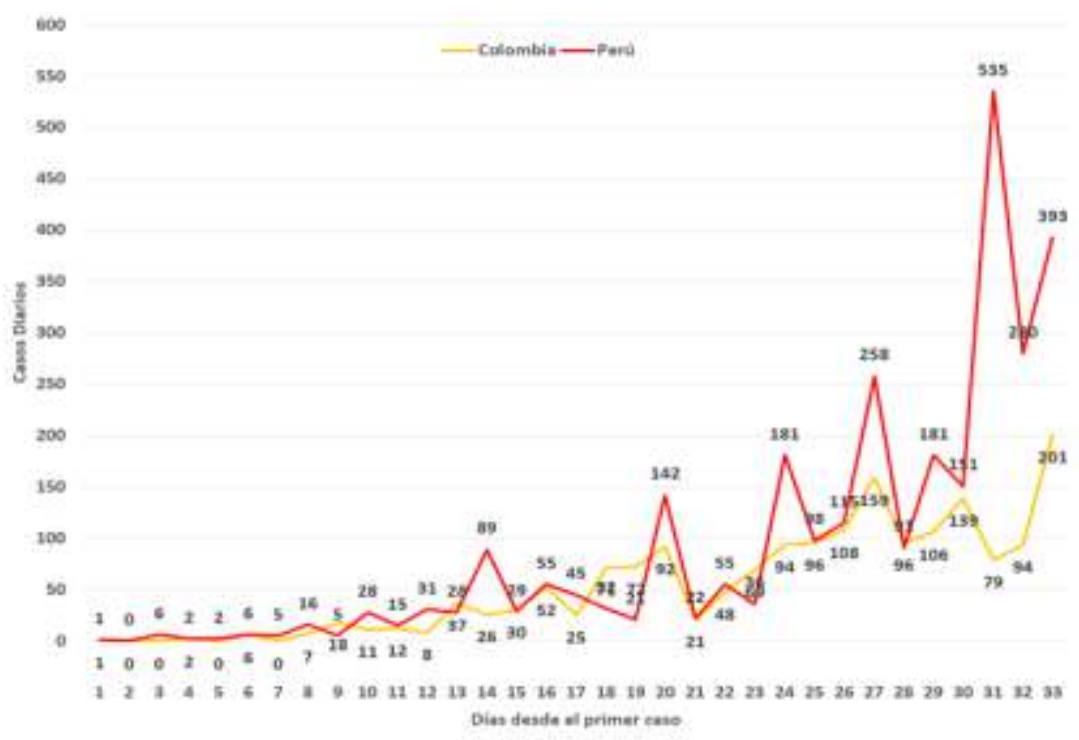

En la región de América Latina, los países deberían unificadamente realizar un manejo integrado de esta pandemia con un abordaje holístico, que va desde la definición estandarizada de casos, que indudablemente en este momento es cambiante, y en consideración a la fase de la epidemia donde nos encontramos, hasta recomendaciones para los trabajadores de la salud en el ámbito clínico. El uso de la mascarilla quirúrgica, fortalecer el lavado de manos, el uso de ropa diferenciada para el hospital, precauciones al llegar a su hogar, entre otras cosas ${ }^{(25)}$.

Entretanto, esperamos que las medidas tomadas, puedan surtir efecto en los países, dado que en SARS funcionaron $^{(39,40)}$, y se considera, que bien aplicadas, en conjunto, podrían también ser de utilidad en el COVID19. La pandemia requiere de mucha unión, esfuerzo, trabajo basado en evidencias científicas e investigación, pero a su vez, prepararse para la fase post-pandemia y el futuro, que requerirá de los profesionales de salud aún más preparación para futuras pandemias que puedan presentarse. Se debe hacer una buena historia clínica, con identificación de síntomas comúnmente reportados, como la fiebre, la tos, síntomas respiratorios, pero también síntomas gastrointestinales, como diarrea, dolor abdominal y vómitos $^{(29,35)}$, así como compromiso neurológico ${ }^{(41,42)}$, incluidas las alteraciones del olfato y del gusto ${ }^{(43)}$, manifestaciones cardiovasculares ${ }^{(44-47)}$, renales ý también cutáneas, recientemente reportadas, como el exantema eritematoso, urticaria y lesiones vesiculosas, con una característica importante que no se presenta con prurito ${ }^{(48,49)}$. Finalmente, el valor diagnóstico de los estudios imagenológicos es también crucial. La radiografía de tórax se convierte en una herramienta de primera línea, evaluando y teniendo en cuenta los hallazgos más frecuentes: opacidades intersticiales, vidrio esmerilado, consolidación basal de predominio periférico y basal. En adición a ello, cuando se posible, la realización de la tomografía axial computarizada (TAC), y en pacientes donde no es posible movilizar al paciente, el uso del ultrasonido pulmonar ${ }^{(50-52)}$.

Finalmente, la educación continuada del médico y los profesionales de la salud, cumplirá un papel vital, en el curso de esta pandemia, especialmente en países latinoamericanos donde el uso racional de recursos, cobra aún más importancia.

Conflictos de interés: El autor niega conflictos de interés.

Financiamiento: Autofinanciado.

\section{REFERENCIAS BIBLIOGRÁFICAS}

1. Gumel AB, Ruan S, Day T, Watmough J, Brauer F, van den Driessche $P$, et al. Modelling strategies for controlling SARS outbreaks. Proc Biol Sci. 2004;271(1554):2223-32.

2. Leung GM, Chung PH, Tsang T, Lim W, Chan SK, Chau P, et al. SARS-CoV antibody prevalence in all Hong Kong patient contacts. Emerg Infect Dis. 2004;10(9):16536.

3. Srikantiah P, Charles MD, Reagan S, Clark TA, Pletz $M W$, Patel PR, et al. SARS clinical features, United States, 2003. Emerg Infect Dis. 2005;11(1):135-8. 
4. Kapoor S, Dhama K. Insight into influenza viruses of animals and humans: Springer; 2014.

5. Mamani OY, Salazar ER, ... Perfil epidemiológico de la Influenza Humana A H1N1 en Cochabamba, Bolivia, gestiones 2009 a 2014: scielo.org. bo; 2015.

6. Gentile A, Paget J, Bellei N, Torres JP, Vazquez C, Laguna-Torres VA, et al. Influenza in Latin America: A report from the Global Influenza Initiative (GII). Vaccine. 2019;37(20):2670-8.

7. World Health Organization. Communicating risk in public health emergencies: a WHO guideline for emergency risk communication (ERC) policy and practice. https://www. who.int/riskcommunication/guidance/download/en/2017 [

8. World Health Organization. One Health. http://www.who.int/features/qa/one-health/en/ 2017[

9. World Health Organization. Novel Coronavirus (2019nCoV) - Situation report - 4 - 24 January 2020. https: / / www. who.int/docs/default source/coronaviruse/situation-reports/20200124sitrep-4-2019-ncov.pdf?sfvrsn=9272d086_2 2020 [

10. World Health Organization. Statement on the meeting of the International Health Regulations (2005) Emergency Committee regarding the outbreak of novel coronavirus (2019-nCoV). https://www.who.int/news-room/detail/23-012020-statement-on-the-meeting-of-theinternational-health-regulations-(2005)-emergencycommittee-regarding-the-outbreak-of-novelcoronavirus-(2019-ncov) 2020 [

11. World Health Organization. Novel Coronavirus (2019$\mathrm{nCoV})$ - Situation report - 7 - 27 January 2020. https: / / www. who.int/docs/defaultsource/coronaviruse/situation-reports/20200127. sitrep-7-2019--ncov.pdf?sfvrsn=98ef79f5_20202020 [

12. World Health Organization. Statement on the second meeting of the International Health Regulations (2005) Emergency Committee regarding the outbreak of novel coronavirus (2019-nCoV). https://www.who.int/news-room/detail/30-012020-statement-on-the-second-meeting-of-theinternational-health-regulations-(2005)-emergencycommittee-regarding-the-outbreak-of-novelcoronavirus-(2019-ncov) 2020 [

13. World Health Organization. Novel Coronavirus (2019nCoV) - Situation report - 10 - 30 January 2020. https: / / www. who.int/docs/default source/coronaviruse/situation-reports/20200130sitrep-10-ncov.pdf?sfvrsn=d0b2e480_2 2020 [

14. World Health Organization. Pneumonia of unknown cause - China. https://www.who.int/csr/don/05january-2020-pneumonia-of-unkown-causechina/en/2020 [

15. World Health Organization;. List of Blueprint priority diseases 2018 [ A vailable from: www.who.int/blueprint/priority-diseases/en/.

16. Musso D, Rodriguez-Morales AJ, Levi JE, Cao-Lormeau VM, Gubler DJ. Unexpected outbreaks of arbovirus infections: lessons learned from the Pacific and tropical America. Lancet Infect Dis. 2018;18(11):e355-e61.

17. Bonilla-Aldana DK, Villamil-Gómez WE, Rabaan AA,
Rodriguez-Morales AJ. Una nueva zoonosis viral de preocupación global: COVID-19, enfermedad por coronavirus 2019. latreia. 2020;33(2):107-10.

18. Dong E, Du H, Gardner L. An interactive web-based dashboard to track COVID-19 in real time. Lancet Infect Dis. 2020.

19. PAHO. Cumulative suspected and confirmed COVID-19 cases reported by countries and territories in the Americas, as of $29 \mathrm{March} 2020$. https://www.paho.org/en/documents/cumulativesuspected-and-confirmed-covid-19-cases-reportedcountries-and-territories-3. 2020.

21. Rodriguez-Morales AJ, MacGregor K, Kanagarajah S, Patel D, Schlagenhauf P. Going global - Travel and the 2019 novel coronavirus. Travel Med Infect Dis. 2020;33:101578.

22. Gallego V, Nishiura H, Sah R, Rodriguez-Morales AJ. The COVID-19 outbreak and implications for the Tokyo 2020 Summer Olympic Games. Travel Med Infect Dis. 2020:101604.

23. Rodriguez-Morales AJ, Sah R, Paniz-Mondolfi A. Should the Holy Week 2020 be cancelled in Latin America due to the COVID-19 pandemic? Travel Medicine and Infectious Disease. 2020:101633.

24. Zambrano LI, Fuentes-Barahona IC, Bejarano-Torres DA, Bustillo C, Gonzales G, Vallecillo-Chinchilla G, et al. A pregnant woman with COVID-19 in Central America. Travel Med Infect Dis. 2020:101639.

25. Rodriguez-Morales AJ, Sánchez-Duque JA, Hernández-Botero S, Pérez-Díaz CE, Villamil-Gómez WE, Méndez CA, et al. Preparación y control de la enfermedad por coronavirus 2019 (COVID-19) en América Latina. Acta Medica Peruana. 2020;37(1):37.

26. Rodriguez-Morales AJ, Gallego V, Escalera-Antezana JP, Mendez CA, Zambrano LI, Franco-Paredes C, et al. COVID-19 in Latin America: The implications of the first confirmed case in Brazil. Travel Med Infect Dis. 2020:101613.

27. Rodriguez-Morales AJ, Suarez JA, Risquez A, DelgadoNoguera L, Paniz-Mondolfi A. The current syndemic in Venezuela: Measles, malaria and more co-infections coupled with a breakdown of social and healthcare infrastructure. Quo vadis? Travel Med Infect Dis. 2019;27:5-8.

28. Navarro J-C, Arrivillaga-Henríquez J, Salazar-Loor J, Rodriguez-Morales AJ. COVID-19 and dengue, coepidemics in Ecuador and other countries in Latin America: Pushing strained health care systems over the edge. Travel Medicine and Infectious Disease. 2020:101656.

29. Escalera-Antezana JP, Lizon-Ferrufino NF, MaldonadoAlanoca A, Alarcon-De-la-Vega G, Alvarado-Arnez LE, Balderrama-Saavedra MA, et al. Clinical features of the first cases and a cluster of Coronavirus Disease 2019 (COVID-19) in Bolivia imported from Italy and Spain. Travel Med Infect Dis. 2020:101653.

30. Bonilla-Aldana DK, Holguin-Rivera Y, Cortes-Bonilla I, Cardona-Trujillo MC, Garcia-Barco A, Bedoya-Arias HA, et al. Coronavirus infections reported by ProMED, February 2000-January 2020. Travel Med Infect Dis. 2020:101575.

31. Millan-Oñate J, Rodríguez-Morales AJ, Camacho- 
Moreno G, Mendoza-Ramírez H, Rodríguez-Sabogal IA, Álvarez-Moreno C. A new emerging zoonotic virus of concern: the 2019 novel Coronavirus (COVID-19). Infectio 2020;24(3):187-92.

32. Rodriguez-Morales AJ, Balbin-Ramon GJ, Rabaan AA, Sah R, Dhama K, Paniz-Mondolfi A, et al. Genomic Epidemiology and its importance in the study of the COVID-19 pandemic. Infez Med. 2020;28(2):139-42.

33. Rodriguez-Morales AJ, Bonilla-Aldana DK, BalbinRamon GJ, Paniz-Mondolfi A, Rabaan A, Sah R, et al. History is repeating itself, a probable zoonotic spillover as a cause of an epidemic: the case of 2019 novel Coronavirus. Infez Med. 2020;28(1):3-5.

34. Sah R, Rodriguez-Morales AJ, Jha R, Chu DKW, Gu H, Peiris M, et al. Complete Genome Sequence of a 2019 Novel Coronavirus (SARS-CoV-2) Strain Isolated in Nepal. Microbiol Resour Announc. 2020;9(11).

35. Rodriguez-Morales AJ, Cardona-Ospina JA, GutierrezOcampo E, Villamizar-Pena R, Holguin-Rivera $Y$, Escalera-Antezana JP, et al. Clinical, laboratory and imaging features of COVID-19: A systematic review and meta-analysis. Travel Med Infect Dis. 2020:101623.

36. Arteaga-Livias FK, Rodriguez-Morales AJ. La comunicación científica y el acceso abierto en la contención de enfermedades: El caso del Coronavirus novel 2019 (2019-nCoV). Rev Peru Investig Salud. 2020;4(1):7-8.

37. Bonilla-Aldana DK, Quintero-Rada K, Montoya-Posada JP, Ramirez-Ocampo S, Paniz-Mondolfi A, Rabaan AA, et al. SARS-CoV, MERS-CoV and now the 2019-novel CoV: Have we investigated enough about coronaviruses? - A bibliometric analysis. Travel Med Infect Dis. 2020;33:101566.

38. Saaavedra-Trujillo $\mathrm{CH}$, et al. Consenso colombiano de atención, diagnóstico y manejo de la infección por SARS-COV-2/COVID-19 en establecimientos de atención de la salud - Recomendaciones basadas en consenso de expertos e informadas en la evidencia. Infectio 2020;24(S3):1-102.

39. Wilder-Smith A, Chiew CJ, Lee VJ. Can we contain the COVID-19 outbreak with the same measures as for SARS? Lancet Infect Dis. 2020.

40. Wilder-Smith A, Freedman DO. Isolation, quarantine, social distancing and community containment: pivotal role for old-style public health measures in the novel coronavirus (2019-nCoV) outbreak. J Travel Med. 2020;27(2).

41. Baig AM. Neurological manifestations in COVID-19 caused by SARS-CoV-2. CNS Neurosci Ther. 2020.
42. Federico A. Brain Awareness Week, CoVID-19 infection and Neurological Sciences. Neurol Sci. 2020.

43. Giacomelli A, Pezzati L, Conti F, Bernacchia D, Siano $M$, Oreni $L$, et al. Self-reported olfactory and taste disorders in SARS-CoV-2 patients: a cross-sectional study. Clin Infect Dis. 2020.

44. Driggin E, Madhavan MV, Bikdeli B, Chuich T, Laracy J, Bondi-Zoccai G, et al. Cardiovascular Considerations for Patients, Health Care Workers, and Health Systems During the Coronavirus Disease 2019 (COVID19) Pandemic. J Am Coll Cardiol. 2020.

45. Guo T, Fan Y, Chen M, Wu X, Zhang L, He T, et al. Cardiovascular Implications of Fatal Outcomes of Patients With Coronavirus Disease 2019 (COVID-19). JAMA Cardiol. 2020.

46. Yang C, Jin Z. An Acute Respiratory Infection Runs Into the Most Common Noncommunicable EpidemicCOVID-19 and Cardiovascular Diseases. JAMA Cardiol. 2020.

47. Zheng YY, Ma YT, Zhang JY, Xie X. COVID-19 and the cardiovascular system. Nat Rev Cardiol. 2020.

48. Wang C, Rademaker M, Baker C, Foley P. COVID-19 and the use of immunomodulatory and biologic agents for severe cutaneous disease: An Australia/New Zealand consensus statement. Australas J Dermatol. 2020.

49. Recalcati S. Cutaneous manifestations in COVID-19: a first perspective. J Eur Acad Dermatol Venereol. 2020.

50. Soldati G, Smargiassi A, Inchingolo R, Buonsenso D, Perrone T, Briganti DF, et al. Proposal for international standardization of the use of lung ultrasound for COVID-19 patients; a simple, quantitative, reproducible method. J Ultrasound Med. 2020.

51. Soldati G, Smargiassi A, Inchingolo R, Buonsenso D, Perrone T, Briganti DF, et al. Is There a Role for Lung Ultrasound During the COVID-19 Pandemic? J Ultrasound Med. 2020.

52. Kalafat E, Yaprak E, Cinar G, Varli B, Ozisik S, Uzun C, et al. Lung ultrasound and computed tomographic findings in pregnant woman with COVID-19. Ultrasound Obstet Gynecol. 2020.

\section{Correspondencia}

Prof. Alfonso J. Rodríguez-Morales, MD, MSc, DTM\&H, FFTM RCPS(Glasg), FACE, HonDSC.

Dirección: Grupo de Investigación Salud Pública e Infección, Facultad de Ciencias de la Salud, Universidad Tecnológica de Pereira, La Julita, Comuna Universidad, Pereira 660003, Risaralda, Colombia.

Correo: arodriguezm@utp.edu.co 